\title{
Las propiedades epigenéticas y anticáncer del ácido valproico
}

\author{
The epigenetic and anticancer properties of valproic acid \\ Gabriela Rebeca Luna-Palencia, ${ }^{*}$ Estefanía Fernández-Navarrete, ${ }^{\ddagger}$ Ismael Vásquez-Moctezuma ${ }^{\ddagger}$
}

* Departamento de Biotecnología, CINVESTAV-IPN, CDMX.

₹ Sección de estudios de

Postgrado e Investigación, Departamento de Morfología, MACISA, ESM-IPN.

\begin{abstract}
RESUMEN
El ácido valproico es un fármaco antiepiléptico que se ha utilizado por más de 50 años. Sin embargo, hace unas décadas se descubrió que posee efectos epigenéticos y este hallazgo permitió proponerlo como fármaco anticáncer. Existe amplia evidencia clínica acumulada en el uso de éste y sus efectos en el humano. El dato clínico y epidemiológico que indica su efecto en el cáncer es la observación de que el uso crónico en un grupo muy extenso de pacientes veteranos de guerra disminuye la frecuencia de cáncer de cabeza y cuello. También existen múltiples ensayos en líneas celulares de humano y modelos animales donde se observa un efecto de detención del ciclo celular, inducción de diferenciación y muerte. En esta revisión se analiza la aplicación de este fármaco en combinación con la quimioterapia.
\end{abstract}

Palabras clave: Ácido valproico, anticáncer, epigenética del cáncer.

\section{ABSTRACT}

Valproic acid is an antiepileptic drug that has been used for more than 50 years. However, a few decades ago, it was discovered that it has epigenetic effects, and this finding allowed it to be proposed as an anticancer drug. There is much accumulated clinical evidence on the use of this and its effects on humans. The epidemiological evidence that indicates its effect on cancer is the observation that the chronic use of valproic acid in a very large group of war veteran patients reduces head and neck cancer frequency. There are also multiple assays on human cell lines and animal models where a cell cycle arrest effect, induction of differentiation and death is observed. This review analyzes the application of this drug in combination with chemotherapy.

Keywords: Valproic acid, anticancer, epigenetics of cancer.
Correspondencia:

Ismael Vásquez-

Moctezuma

E-mail:

g17isma65@gmail.com

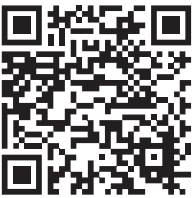

\section{INTRODUCCIÓN}

Al igual que con fármacos como la aspirina, que adicionalmente a su efecto analgésico posee otras propiedades como la capacidad anticoagulante, o como para las estatinas que aparte de disminuir el colesterol son antiinflamato- rias, ${ }^{1,2}$ el ácido valproico (AVP) inicialmente se elaboró como un análogo del ácido valérico (obtenido de la planta Valeriana officinalis) y en 1964 se reportó su efecto antiepiléptico. ${ }^{3-5}$ Este medicamento se utiliza en enfermedades como la epilepsia, migraña, hiperactividad, trastorno bipolar y por déficit de atención. ${ }^{5}$ Esta

Citar como: Luna-Palencia GR, Fernández-Navarrete E, Vásquez-Moctezuma I. Las propiedades epigenéticas y anticáncer del ácido valproico. Rev Mex Mastol. 2020; 10 (2): 54-62. https://dx.doi.org/10.35366/97715 
revisión se enfoca en los usos del AVP como un fármaco inhibidor de las desacetilasas de histona (iHDACs) y con efecto sensibilizador en los fármacos de la quimioterapia tradicional. ${ }^{6}$

\section{EL ORIGEN EPIGENÉTICO DEL CÁNCER HUMANO}

El cáncer es una de las principales causas de muerte en el mundo, sólo después de las provocadas por las complicaciones de la diabetes mellitus y de la hipertensión arterial, por lo que se considera un problema de salud pública. Las neoplasias malignas se pueden comportar de modo distinto, tener diferente pronóstico, y evolucionar cada una de manera muy particular; sin embargo, en todas ellas existe un común denominador: la pérdida de la capacidad celular para controlar su proliferación, invadiendo territorios reservados para otras células (metástasis). Esta falta de regulación de la proliferación se debe a las alteraciones en genes supresores de tumores y en protooncogenes que inducen el desarrollo de células malignas. Los cambios genéticos y epigenéticos se van acumulando y combinando con el paso del tiempo, lo que lleva a la transformación neoplásica.?

\section{MECANISMOS EPIGENÉTICOS EN EL CÁNCER}

Los mecanismos epigenéticos incluyen tres procesos, en primer lugar, cambios en las proteínas de histona por medio de modificaciones postraduccionales como la acetilación y desacetilación, forforilación, sumoilación, metilación. En segundo lugar, metilación de las regiones ricas en citosinas (CpG) llamadas «islas CpG» agrupadas en algunas secuencias del ADN de las regiones promotoras de la mitad de los genes humanos, y que metiladas inhiben la expresión de éstos. En tercer lugar, el mecanismo de bloqueo de ARNs mensajeros por moléculas de microARNs (miARNAs). De manera muy general, los procesos epigenéticos actúan en el cáncer por medio de la hipermetilación de regiones promotoras y la desacetilación de histonas que puede apagar genes supresores de tumores y los cambios en la expresión de ciertos miARNs que pueden funcionar con oncogenes. A diferencia de las alteraciones genéticas que son estructurales e irreversibles como las mutaciones, las epigenéticas son funcionales y por lo tanto, son reversibles. ${ }^{8,9}$

\section{DESACETILASAS DE HISTONAS (HDACS) Y EL CÁNCER HUMANO}

En el núcleo celular, el ADN se enrolla y empaqueta alrededor de estructuras heterotetraméricas constituidas por cuatro proteínas histonas $(\mathrm{H} 2 \mathrm{~A}, \mathrm{H} 2 \mathrm{~B}, \mathrm{H} 3$ y $\mathrm{H} 4)$ que forman el nucleosoma, que cuando se condensan integran la cromatina. ${ }^{9}$ Cada histona del nucleosoma tiene una «cola» en el extremo amino-terminal rica en el aminoácido básico lisina, esta prolongación de la histona se extiende por fuera del nucleosoma y la accesibilidad del ADN es en parte controlada por modificaciones de esta «cola» peptídica. ${ }^{10-12} \mathrm{El}$ proceso de acetilación de histonas y de diferentes proteínas citoplasmáticas se controla mediante dos tipos de enzimas: las acetiltransferasas de histonas (HATs) y las desacetilasas de histonas (HDACs). ${ }^{13}$ Las HATs transfieren grupos acetilo, que provienen de la acetil Co-A, a los residuos de lisina de las colas de histonas; esta adición del grupo acetilo neutraliza la carga positiva de los residuos de la lisina de las histonas, disminuyendo la atracción con el ADN (cargado negativamente), permitiendo el acceso de factores de transcripción y de la enzima ARN polimerasa entre otros conjuntos de proteínas reguladoras. En contraste, las HDACs remueven los grupos acetilo, lo que por lo general disminuye la accesibilidad de factores de transcripción y de la ARN polimerasa. ${ }^{12,13}$

La mayoría de las HATs humanas participan como coactivadores de la transcripción, la función más estudiada de este grupo de proteínas es la acetilación de las colas de histonas. También se conoce que estas enzimas poseen una función citoplásmica al acetilar proteínas en complejos macromoleculares que actúan corregulando funciones celulares importantes como la expresión de genes por medio de factores de transcripción, el ciclo celular, a proteínas que participan en el splicing, procesos de transporte y nucleación de actina. ${ }^{14}$ Entre las proteínas del citoplasma que son acetiladas y desacetiladas para regular ciertos aspectos de 
su función están la tubulina, p53, Hsp90, Bcl-2 y Ku70. ${ }^{14,15}$

\section{LA FAMILIA DE LAS DESACETILASAS DE HISTONAS}

Se conocen 11 HDACs en el humano y se dividen en cuatro clases, las de clase I, II, III y IV. ${ }^{14}$ Se han clasificado de acuerdo a su homología con las HDACs de levadura, localización subcelular y por su actividad enzimática. Son proteínas de 40-55 kDa que depende del $\mathrm{Zn}^{+}$ como cofactor y se expresan de manera ubicua en los tejidos humanos e incluyen a las HDACs de clase I, compuesta por las HDAC1, 2, 3 y 8 que son homólogas a las proteínas de levadura y de localización citoplasmática, las HDACs 4,5,7 y 9 son de la clase lla y se encuentran tanto en el núcleo como en el citoplasma, las de clase Ilb en el citoplasma incluyen la HDAC6 (ésta no desacetila histonas) y HDAC 9, finalmente la HDAC 11 se localiza en el citoplasma. Las HDACs de clase III se conocen como sirtuinas y ocupan el NAD como cofactor. ${ }^{14-16}$

La función de estas enzimas se ha estudiado principalmente en líneas celulares de cáncer humano y se orienta a los mecanismos como la proliferación donde son importantes las HDAC1, HDAC2, HDAC3 y HDAC8; en la apoptosis destacan la HDAC1 y la HDAC2. Con respecto a la resistencia a la quimioterapia es importante la HDAC1; para la diferenciación, las HDAC3, HDAC4, HDAC5 y HDAC8; en el caso de la angiogénesis, las HDAC4, HDAC6, HDAC7 y HDAC10; y finalmente para la migración, la HDAC6. ${ }^{17-19}$ La sobreexpresión de las HDACs se ha visto relacionada con el proceso de tumorigénesis en diferentes tipos de cáncer (Tabla 1).

\section{LOS INHIBIDORES DE LAS DESACETILASAS DE HISTONAS (IHDACS) Y SU APLICACIÓN EN EL CÁNCER HUMANO}

Los iHDACs aplicados a líneas celulares transformadas generan diferenciación, detención del ciclo celular e inducen apoptosis. Se ha observado que inhiben el crecimiento tumoral en modelos animales; y muestran actividad antitumoral en ensayos clínicos controlados. ${ }^{20}$
Además, son capaces de activar el mecanismo de muerte celular llamado autofagia, generan especies reactivas de oxígeno, inhiben la proteína de choque térmico Hsp90 e interrumpen la vía del agresoma. ${ }^{14}$

En la actualidad, y con las evidencias acumuladas de que el desarrollo del cáncer se relaciona con hipoacetilación de las histonas, los fármacos con propiedades epigenéticas del tipo inhibidores de las deacetilasas de histonas o iHDACs se han comenzado a introducir en el tratamiento contra el cáncer. Parte del mecanismo de acción de estos fármacos se explica debido a la inhibición de las enzimas deacetilasas, lo que lleva a un aumento en la acetilación de las colas de histonas. Este cambio postraduccional en las histonas reactiva la transcripción de genes supresores de tumores, entre otros, dando la posibilidad de revertir el proceso maligno. ${ }^{14}$ En estudios clínicos los iHDACs han mostrado resultados aceptables en el tratamiento de neoplasias hematológicas, por lo que la FDA ha aprobado el suberolinida o SAHA para el tratamiento del linfoma cutáneo de células $\mathrm{T}$, además de la displasia de médula ósea y la romidepsina para el linfoma de células T. (Sin embargo, en tumores sólidos los resultados son variables y no tan espectaculares. ${ }^{21}$ Cabe mencionar que las aplicaciones para los inhibidores de las HDACs podrían ser útiles en otras enfermedades tan diversas como infecciones virales crónicas, obesidad, síndrome metabólico y diabetes mellitus 2 , entre otras. ${ }^{9}$

\section{DETENCIÓN DEL CICLO CELULAR POR COMPUESTOS EPIGENÉTICOS}

Los mecanismos de los efectos anticáncer de los inhibidores de HDAC no son uniformes; pueden ser diferentes $y$ depender de un tipo de cáncer, del inhibidor de las HDAC y de su dosis así como de otros factores.

Como se entiende, detener el ciclo celular en la célula de cáncer sería importantísimo para evitar el crecimiento tumoral, esto es precisamente lo que hacen los inhibidores de HDACs como el ácido valproico. El principal mecanismo de detención del ciclo celular es el aumento de la expresión de genes como CDKN1A (que codifica para la proteína p21 


\begin{tabular}{|c|c|c|c|}
\hline HDAC & Localización & Alteración en el cáncer & Tumor \\
\hline \multicolumn{4}{|c|}{ Clase I } \\
\hline HDAC1 & Núcleo & Sobreexpresión, baja expresión & Esófago, colon, próstata, $\mathrm{CTCL}$ \\
\hline HDAC2 & Núcleo & Sobreexpresión/mutación & Próstata, colon, gástrico, $\mathrm{CTCL}$ \\
\hline HDAC3 & Núcleo & Sobreexpresión & Próstata, colon \\
\hline HDAC8 & Núcleo & Sobreexpresión & Colon \\
\hline \multicolumn{4}{|c|}{ Clase Ila } \\
\hline HDAC4 & Núcleo/citoplasma & Sobreexpresión/baja expresión & Próstata, colon, mama \\
\hline HDAC5 & Núcleo/citoplasma & Baja expresión & Colon, AML \\
\hline HDAC7 & Núcleo/citoplasma & Sobreexpresión & Colon \\
\hline HDAC9 & Núcleo/citoplasma & Sobreexpresión/bajaexpresión & Meduloblastoma \\
\hline & & & Astrocitoma \\
\hline \multicolumn{4}{|c|}{ Clase Ilb } \\
\hline HDAC6 & Citoplasma & Sobreexpresión & Mama, AML, CTLT \\
\hline HDAC10 & Citoplasma & Sobreexpresión & Carcinoma hepatocelular \\
\hline \multicolumn{4}{|c|}{ Clase III } \\
\hline HDAC11 & Núcleo/citoplasma & Sobreexpresión & Mama \\
\hline
\end{tabular}

el inhibidor de quinasa dependiente de ciclina) en líneas celulares de cáncer. ${ }^{22-24} \mathrm{Su}$ producto bloquea la formación de dímeros a partir de ciclinas y quinasas dependientes de ciclina (CDK) y detiene el ciclo celular. Además, es capaz de inhibir la diferenciación celular. ${ }^{23,24}$ La expresión del gen p21 está controlada por la proteína p53 que interactúa con el promotor de este gen, compitiendo con HDAC1, y esto disminuye la expresión de p21. ${ }^{25}$ Posterior al tratamiento con inhibidores de las HDAC, la enzima HDAC1 se libera del Sp1 (un factor de transcripción específico del promotor), lo que aumenta la expresión de p21 (freno del ciclo celular). La inhibición de HDAC, a nivel del citoplasma, aumenta la acetilación de la proteína p53, lo que resulta en un incremento de su vida media, mejorando así la interacción con el promotor p21. ${ }^{26,27}$ Por último, los niveles de p21 se incrementan, mediando así la detención del ciclo celular y la apoptosis. ${ }^{23,28,29}$
Los inhibidores de HDAC también pueden detener la expresión de genes que codifican ciclina D y ciclina A dando como resultado la ausencia de actividades de las correspondientes quinasas, CDK2 y CDK4. ${ }^{24,30}$

\section{INDUCCIÓN DE LAAPOPTOSIS POR AGENTES EPIGENÉTICOS}

Los inhibidores de las HDAC inducen la apoptosis en las células cancerígenas por medio de la regulación de genes proapoptóticos y antiapoptóticos. ${ }^{31-33}$ Los mecanismos por los que diferentes inhibidores de HDAC inducen la apoptosis incluyen la activación de vías apoptóticas tanto extrínsecas como intrínsecas. La activación de la vía apoptótica de tipo extrínseco por los iHDAC se demostró en experimentos in vitro. Se ha observado que estos compuestos influyen en los receptores de muerte TRAIL (ligando inductor de apoptosis relacionado con TNF), DR5 (receptor de muerte 5), Fas 
(superfamilia 6 de TNF), TNF (factor de necrosis tumoral) y ligandos relacionados con el TNF Fas-L. ${ }^{32,34-36}$ Los inhibidores de HDAC también activan la vía apoptótica intrínseca, regulan la transcripción de genes proapoptóticos como Bid (proteína agonista de muerte del dominio que interactúa con BH3), Bad (agonista de la proteína de muerte celular asociado a Bcl-2) y Bim. $22,33,37,38$

Además de estos efectos en la expresión génica, los inhibidores de HDAC aumentan las especies reactivas de oxígeno (ROS) que pueden inducir apoptosis en células leucémicas (Jurkat, ML-1, U937, HL-60, K-562, CEMCCRF. $^{37,39,40}$ El AVP induce la apoptosis de manera más eficaz en condiciones hipóxicas y supera la resistencia inducida por hipoxia al cisplatino (CDDP) en las células derivadas de neuroblastoma de alto riesgo UKF-NB-3 y sublínea resistente a CDDP, probablemente por inducción de la degradación de HIF-1 $\alpha .{ }^{41,42}$

\section{LA INDUCCIÓN DE LA MUERTE POR AUTOFAGIA}

La acetilación de muchas proteínas relacionadas con la autofagia, como el producto de los genes relacionados con la autofagia (ATG), está regulada por el equilibrio entre HAT y HDAC. ${ }^{43}$ La autofagia también puede regularse mediante la acetilación de factores de transcripción como FOXO. ${ }^{44}$ Diferentes HDAC influyen en la actividad autofágica mediante mecanismos diversos. La HDAC6 induce la autofagia cuando se deteriora el sistema de ubiquitina-proteasoma (UPS). La inhibición o eliminación de HDAC1 en células HeLa promueve la formación de vacuolas autofágicas. ${ }^{45}$ La sirtuina SIRT1 forma un complejo con componentes de la maquinaria de autofagia (Atg5, -7 y -8) y estimula la muerte por este mecanismo. ${ }^{46}$

\section{EL ÁCIDO VALPROICO Y CAPACIDAD PARA MODIFICAR LOS MECANISMOS EPIGENÉTICOS}

Desde 1985 se descubrió la capacidad del AVP para inhibir la proliferación celular en el neuroblastoma murino y en células de glioma; también se ha encontrado que la exposición continua a AVP induce la diferenciación de varias líneas celulares y genera apoptosis. En el año 2001, un grupo alemán descubrió que el AVP, un fármaco utilizado en el control de la epilepsia, tiene actividad como iHDAC. ${ }^{47} \mathrm{El}$ AVP se considera un fármaco inhibidor de las HDACs que causa hiperacetilación de las colas $\mathrm{N}$-terminales de las histonas $\mathrm{H} 3$ y $\mathrm{H} 4$ in vitro e in vivo. ${ }^{47}$ Se ha clasificado a este fármaco como un inhibidor selectivo de las HDACs clase I. ${ }^{16,19,48}$

\section{AVP COMO POSIBLE AGENTE INHIBIDOR DE LA HDAC8, ESTUDIOS DE DOCKING}

Se desconoce el mecanismo por el cual el AVP inhibe las HDACs; sin embargo, en un estudio in silico, en el que se utilizaron 14 estructuras halladas en el Protein Data Bank que pertenecen a la HDAC8, una isoforma clase I y utilizando al AVP como ligando, se encontró que hay dos sitios de unión: el sitio catalítico (CS) y el canal hidrofóbico del sitio activo (HASC). El grupo carboxilo del AVP interactúa con el CS, formando puentes de hidrógeno con residuos específicos (H142-143 y Y306) e interacciones electrostáticas con el átomo de $\mathrm{Zn}^{+}$. Por otro lado, el HASC es un canal hidrofóbico donde se piensa que se libera el acetato después de la reacción. Los resultados de las simulaciones por acoplamiento molecular muestran que el AVP tiene mayor afinidad por el HASC, esto sugiere que este canal es el sitio de unión del AVP, lo que hace suponer que existe un mecanismo donde esta interacción del AVP con el HASC bloquea la liberación del acetato e inhibe la actividad catalítica de la HDAC. Este análisis in silico es muy importante, ya que el CS ha sido el blanco principal para el diseño de ligandos, pero como existen pocas diferencias estructurales entre todos los CS de las HDACs, la inhibición resultante no es específica como es el caso del SAHA. ${ }^{49}$

\section{LOS IHDACs APROBADOS PARA TERAPIA EN HUMANOS}

En el año 2006, la Administración Federal de Drogas de Estados Unidos (FDA) autorizó el uso de la suberolinida de ácido hidroxámico o SAHA como el primer fármaco inhibidor de 
las HDACs para tratar pacientes con linfoma cutáneo de células $\mathrm{T}(\mathrm{CTCL})$ que remiten o que no responden al tratamiento. ${ }^{50}$ También se han observado buenos resultados en linfoma folicular y en el de zona marginal. ${ }^{51}$ Sin embargo, en estudios clínicos fase II con suberolinida de ácido hidroxámico (SAHA) para el tratamiento de tumores de cabeza y cuello es tolerada por los pacientes, pero las respuestas a esta terapia no son significativas. ${ }^{52}$ En 2009, la FDA aprobó el uso de la romidepsina, un péptido cíclico, para el tratamiento del CTCL y recientemente se aprobó para el tratamiento de pacientes diagnosticados con linfoma de células $T$ periféricas (PTCL). ${ }^{14}$

\section{APLICACIONES DEL ÁCIDO VALPROICO EN EL CÁNCER HUMANO}

Se sabe que el AVP es capaz de hacer a las células de cáncer más sensibles a la quimioterapia y radioterapia. Este fármaco se ha usado en la terapia del cáncer humano como monoterapia o combinado con agentes desmetilantes, quimioterapia y moduladores inmunitarios. ${ }^{53}$ En un estudio clínico de fase II se observó que utilizado como monoterapia y aplicado en carcinoma neuroendocrino induce la expresión de la proteína Notch I, que es un supresor de tumores. En el estudio participaron ocho pacientes, uno respondió de manera parcial y cinco evolucionaron a una patología estable. ${ }^{54} \mathrm{En}$ estudios realizados in vitro en células derivadas de neoplasias mieloides mostraron un efecto de inducción de apoptosis y diferenciación de las células leucémicas, lo que ha estimulado el uso del AVP como monoterapia o combinado en la leucemia mieloide aguda (AML) y en el síndrome mielodisplásico (MDS). En otro estudio clínico controlado de fase II en el cual participaron 75 individuos tratados con el AVP en combinación con el ácido all-trans retinoico se reportó que 18 pacientes (24\%) tuvieron respuestas consideradas adecuadas. En otros trabajos se usó la 5-azacitidina más el AVP, resultando en una respuesta superior a la terapia convencional en estudios fases I, II y III en pacientes mayores afectados con AML y MDS. ${ }^{55,56}$ En otro trabajo clínico fase II en el cual se utilizó el AVP asociado al ácido all-trans retinoico y la 5-azacitidina que se aplicaron a personas con
AML y con MDS, se obtuvo respuesta en $23 \%$ de los pacientes y una supervivencia de 12.4 meses. ${ }^{57}$ Se han hecho combinaciones entre agentes que dañan el ADN con el valproico, un ejemplo de esto es el trabajo en el que se asoció con la epirubicina (inhibidor de las enzimas topoisomerasas tipo II) dando respuesta en nueve de los 44 pacientes. ${ }^{58}$ En un estudio fase I-II se combinó al AVP con 5-fluorouracilo, epirubicina y ciclofosfamida en un grupo de 15 mujeres con cáncer de mama; en 64\% de éstas se observó una toxicidad aceptable. ${ }^{53} \mathrm{En}$ otra publicación clínica de estudios fase I/II de pacientes con melanoma metastásico se utilizó AVP en combinación con la karenitecina, un inhibidor de la topoisomerasa l; el resultado fue estabilización de la enfermedad en $47 \% .{ }^{59}$ En un trabajo de tipo aleatorizado, fase III con 36 pacientes afectados de cáncer cervicouterino, la asociación entre hidralazina, AVP, cisplatino y topotecán generó mejoría en la supervivencia. ${ }^{60}$ Otro trabajo in vitro demostró que el AVP aumenta la acetilación de la histona H3. En un análisis retrospectivo de una cohorte de 439,628 adultos mayores tratados con AVP por diferentes padecimientos neurológicos (trastorno bipolar, migraña, epilepsia) se observó menor frecuencia de carcinoma de cabeza y cuello relacionado con el tabaquismo en 26,911 individuos que utilizaron el AVP crónicamente. ${ }^{61}$

En México, el grupo de Dueñas-González está realizando estudios clínicos en fase III, utilizando el AVP como iHDAC en la terapia contra el cáncer cervical, al asociar este medicamento a la hidralazina, un fármaco antihipertensivo con propiedades desmetilantes (TRANSKRIPT $\left.{ }^{\circledR}\right) .{ }^{62}$

\section{EL AVP HA SERVIDO DE BASE PARA LA CREACIÓN DE MOLÉCULAS ANTICÁNCER}

El grupo del doctor José Correa-Basurto se ha enfocado al diseño, modelado molecular, síntesis y análisis biológicos de profármacos derivados del AVP. Recientemente se han sintetizado moléculas que acoplan al AVP con diferentes aminoácidos como la prolina y el glutámico que en ensayos de la línea celular de cáncer cervicouterino HeLa y sarcoma disminuyen la viabilidad de las mismas. ${ }^{63-65}$ 


\section{CONCLUSIONES}

En la actualidad se conoce que las células madre del cáncer humano y el microambiente tumoral son en buena proporción los que controlan el desarrollo y resistencia de las neoplasias. Con la idea de eliminar estas células malignas se han hecho esfuerzos por aislar a esta población de células madre del cáncer por medio de marcadores de membrana, lo que sólo se ha logrado de manera adecuada en células leucémicas. En el caso de tumores sólidos, hasta el momento no se ha obtenido una población pura de células madre del cáncer para eliminarlas por medio de anticuerpos específicos o drogas selectivas. El hecho de utilizar fármacos inhibidores de las HDACs nos abre la posibilidad de que estas células madre inmaduras avancen en los estadios de maduración y diferenciación, expresen genes de marcadores de superficie o proteínas susceptibles de ser un blanco específico para las drogas y anticuerpos monoclonales terapéuticos.

El concepto de eliminar totalmente las células en el individuo se ha modificado en las últimas décadas. Por desgracia la quimioterapia y radioterapia son las únicas medidas terapéuticas que han mostrado resultados para algunos tipos de cáncer. Pero, como ya se ha mencionado, con un alto costo para el organismo humano por la toxicidad, lesiones oxidativas y muerte celular que se genera en los tejidos sanos. La terapia biológica, hormonal y de inhibidores de quinasas ha cambiado un poco ese concepto para dar paso al control adyuvante crónico de procesos malignos combinados con los procedimientos tradicionales quirúrgicos y de otro tipo. Los medicamentos epigenéticos como el ácido valproico nos ofrecen la posibilidad de revertir algunas características malignas de las células de cáncer, con la esperanza de que algún día estas células malignas sean el blanco de fármacos de la quimioterapia tradicional o de la terapia biológica, utilizados en dosis pequeñas, mínimamente tóxicas y a bajo costo.

\section{BIBLIOGRAFÍA}

1. Awtry EH, Loscalzo J. Aspirin. Circulation. 2000; 101 (10): 1206-1218.

2. Abeles AM, Pillinger MH. Statins as antiinflammatory and immunomodulatory agents: a future in rheumatologic therapy? Arthritis Rheum. 2006; 54 (2): 393-407.
3. Löscher W. Basic pharmacology of valproate. A review after 35 years of clinical use for the treatment of epilepsy. CNS Drugs. 2002; 16 (10): 669-694.

4. Frazee LA, Foraker KC. Use of intravenous valproic acid for acute migraine. Ann Pharmacother. 2008; 42 (3): 403-407.

5. Rosenberg G. The mechanisms of action of valproate in neuropsychiatric disorders: can we see the forest for the trees? Cell Mol Life Sci. 2007; 64 (16): 2090-2103.

6. Luna-Palencia GR, Correa-Basurto J, Vásquez-Moctezuma I. El ácido valproico como agente sensibilizador al tratamiento anticáncer. Gac Med Mex. 2018; 155 (4): 417-422.

7. Hanahan D, Weinberg RA, Hallmarks of cancer: the next generation. Cell. 2011; 144 (5): 646-674

8. Dawson MA, Kouzarides T. Cancer epigenetics: from mechanism to therapy. Cell. 2012; 150 (1): 12-27.

9. Henikoff S, Greally JM. Epigenetics, cellular memory and gene regulation. Curr Biol. 2016; 26 (14): R644R648.

10. Tessarz P, Kouzarides T. Histone core modifications regulating nucleosome structure and dynamics. Nature Reviews Molecular Cell Biology. 2014; 15 (11): $703-$ 708.

11. Mersfelder EL, Parthun MR. The tale beyond the tail: histone core domain modifications and the regulation of chromatin structure. Nucleic Acids Res. 2006; 34 (9): 2653-2662.

12. Seto $E$, Yoshida M. Erasers of histone acetylation: the histone deacetylase enzymes. Cold Spring Harb Perspect Biol. 2014; 6 (4): a018713.

13. Choudhary C, Kumar C, Gnad F, Nielsen ML, Rehman $\mathrm{M}$, Walther TC et al. Lysine acetylation targets protein complexes and co-regulates major cellular functions. Science 2009; 325 (5942): 834-840.

14. Robey RW, Chakraborty AR, Basseville A, Luchenko V, Bahr J, Zhan Z et al. Histone deacetylase inhibitors: emerging mechanisms of resistance. Mol Pharm. 2011; 8 (6): 2021-2031.

15. Delcuve GP, Khan DH, Davie JR. Roles of histone deacetylases in epigenetic regulation: emerging paradigms from studies with inhibitors. Clin Epigenetics. 2012; 4 (1): 5.

16. Sanaei M, Kavoosi F., Histone deacetylases and histone deacetylase inhibitors: molecular mechanisms of action in various cancers. Adv Biomed Res. 2019; 8: 63

17. De Ruijter AJM, Van Gennip AH, Caron HN, Kemp S, Van Kuilenburg AB. Histone deacetylases (HDACs): characterization of the classical HDAC family. Biochem. J. 2003; 370 (Pt 3): 737-749.

18. Dejligbjerg M, Grauslund M, Litman T, Collins L, Qian $X$, Jeffers $M$ et al. Differential effects of class I isoform histone deacetylase depletion and enzymatic inhibition by belinostat or valproic acid in HeLa cells. Mol Cancer. 2008; 7: 70 .

19. Witt O, Deubzer HE, Lodrini M, Milde T, Oehme I. Targeting histone deacetylases in neuroblastoma. Curr Pharm Des. 2009; 15 (4): 436-447.

20. Fantin VR, Richon VM. Mechanisms of resistance to histone deacetylase inhibitors and their therapeutic implications. Clin Cancer Res. 2007; 13 (24): 7237 7242. 
21. Mercurio C, Minucci S, Pelicci PG. Histone deacetylases and epigenetic therapies of hematological malignancies. Pharmacol Res. 2010; 62 (1): 18-34.

22. Vrana JA, Decker RH, Johnson CR, Wang Z, Jarvis WD, Richon VM et al. Induction of apoptosis in U937 human leukemia cells by suberoylanilide hydroxamic acid (SAHA) proceeds through pathways that are regulated by $\mathrm{BCl}-2 / \mathrm{BCl}-\mathrm{XL}$, c-Jun, and p21CIP1, but independent of p53. Oncogene 1999; 18 (50): 7016-7025.

23. Richon VM, Sandhoff TW, Rifkind RA, Marks PA. Histone deacetylase inhibitor selectively induces p21WAF1 expression and gene-associated histone acetylation. Proc Natl Acad Sci USA. 2000; 97 (18): 10014-10019.

24. Sandor V, Senderowicz A, Mertins S, Sackett D, SausviIle E, Blagosklonny MV et al. P21-dependent G1arrest with downregulation of cyclin D1 and upregulation of cyclin E by the histone deacetylase inhibitor FR901228. Br J Cancer. 2000; 83 (6): 817-825.

25. Ocker M, Schneider-Stock R. Histone deacetylase inhibitors: Signalling towards p21cip1/waf1. Int J Biochem Cell Biol. 2007; 39 (7-8): 1367-1374.

26. Gius D, Cui H, Bradbury CM, Cook J, Smart DD, Zhao $S$ et al. Distinct effects on gene expression of chemical and genetic manipulation of the cancer epigenome revealed by a multimodality approach. Cancer Cell 2004; 6 (4): 361-371.

27. Zhao Y, Lu S, Wu L, Chai G, Wang H, Chen Y et al. Acetylation of p53 at lysine 373/382 by the histone deacetylase inhibitor depsipeptide induces expression of p21(Waf1/Cip1). Mol Cell Biol. 2006; 26 (7): 27822790

28. Mahyar-Roemer M, Roemer K. p21 Waf1/Cip1 can protect human colon carcinoma cells against p53dependent and p53-independent apoptosis induced by natural chemopreventive and therapeutic agents. Oncogene. 2001; 20 (26): 3387-3398.

29. Suzuki T, Yokozaki, H, Kuniyasu H, Hayashi K, Naka $\mathrm{K}$, Ono $\mathrm{S}$ et al. Effect of trichostatin A on cell growth and expression of cell cycle- and apoptosis-related molecules in human gastric and oral carcinoma cell lines. Int J Cancer. 2000; 88 (6): 992-997.

30. Qiu L, Burgess A, Fairlie DP, Leonard H, Parsons PG, Gabrielli BG. Histone deacetylase inhibitors trigger a G2 checkpoint in normal cells that is defective in tumor cells. Mol Biol Cell. 2000; 11 (6): 2069-2083.

31. Kim HJ, Bae SC. Histone deacetylase inhibitors: Molecular mechanisms of action and clinical trials as anti-cancer drugs. Am J Transl Res. 2011; 3 (2): 166-179.

32. Minucci S, Pelicci PG. Histone deacetylase inhibitors and the promise of epigenetic (and more) treatments for cancer. Nat Rev Cancer. 2006; 6 (1): 38-51.

33. Miller CP, Singh MM, Rivera-Del Valle N, Manton CA, Chandra J. Therapeutic strategies to enhance the anticancer efficacy of histone deacetylase inhibitors. J Biomed. Biotechnol. 2011; 2011: 514261.

34. Fulda S. Modulation of TRAIL-induced apoptosis by HDAC inhibitors. Curr Cancer Drug Targets 2008; 8 (2): 132-140.

35. Kwon SH, Ahn SH, Kim YK, Bae GU, Yoon JW, Hong S et al. Apicidin, a Histone Deacetylase Inhibitor, induces apoptosis and Fas/Fas ligand expression in human acute promyelocytic leukemia cells. J Biol Chem. 2002; 277 (3): 2073-2080.
36. Nebbioso A, Clarke N, Voltz E, Germain E, Ambrosino C, Bontempo P et al. Tumor-selective action of HDAC inhibitors involves TRAIL induction in acute myeloid leukemia cells. Nat Med. 2005; 11 (1): 77-84.

37. Ruefli AA, Ausserlechner MJ, Bernhard D, Sutton VR, Tainton KM, Kofler R et al. The histone deacetylase inhibitor and chemotherapeutic agent suberoylanilide hydroxamic acid (SAHA) induces a cell-death pathway characterized by cleavage of Bid and production of reactive oxygen species. Proc Natl Acad. Sci USA. 2001; 98 (19): 10833-10838.

38. Zhao Y, Tan J, Zhuang L, Jiang X, Liu, ET, Yu Q. Inhibitors of histone deacetylases target the Rb-E2F1 pathway for apoptosis induction through activation of proapoptotic protein. Bim Proc Natl Acad Sci USA. 2005; 102 (44): 16090-16095.

39. Gao S, Mobley A, Miller C, Boklan, J, Chandra J. Potentiation of reactive oxygen species is a marker for synergistic cytotoxicity of MS-275 and 5-azacytidine in leukemic cells. Leuk Res. 2008; 32 (5): 771-780.

40. Rosato RR, Almenara JA, Grant S. The histone deacetylase inhibitor MS-275 promotes differentiation or apoptosis in human leukemia cells through a process regulated by generation of reactive oxygen species and induction of p21CIP1/WAF11. Cancer Res. 2003; 63 (13): 3637-3645

41. Cipro Š, H $\square$ ebačková J, Hrab】ta J, Poljaková J, Eckschlager T. Valproic acid overcomes hypoxia-induced resistance to apoptosis. Oncol Rep. 2012; 27 (4): 1219-1226.

42. Jeong JW, Bae MK, Ahn MY, Kim SH, Sohn TK, Bae $\mathrm{MH}$ et al. Regulation and destabilization of HIF- $1 \alpha$ by ARD1-mediated acetylation. Cell. 2002; 111 (5): 709-720.

43. Zhang J, Zhong Q. Histone deacetylase inhibitors and cell death. Cell Mol Life Sci. 2014; 71 (20): 3885-3901.

44. Brunet A, Sweeney LB, Sturgill JF, Chua KF, Greer $\mathrm{PL}$, Lin $\mathrm{Y}$ et al. Stress-dependent regulation of FOXO transcription factors by the SIRT1 deacetylase. Science 2004; 303 (5666): 2011-2015.

45. Oh M, Choi IK, Kwon HJ. Inhibition of histone deacetylase1 induces autophagy. Biochem Biophys Res Commun. 2008; 369 (4): 1179-1183.

46. Lee IH, Cao L, Mostoslavsky R, Lombard DB, Liu J, Bruns NE et al. A role for the NAD-dependent deacetylase Sirt1 in the regulation of autophagy. Proc Natl Acad Sci USA. 2008; 105 (9): 3374-3379.

47. Göttlicher M, Minucci S, Zhu P, Krämer OH, Schimpf A, Giavara S, Sleeman JP et al.. Valproic acid defines a novel class of HDAC inhibitors inducing differentiation of transformed cells. EMBO J. 2001; 20 (24): 69696978.

48. Khan N, Jeffers M, Kumar S, Hackett C, Boldog F, Khramtsov N et al. Determination of the class and isoform selectivity of small-molecule histone deacetylase inhibitors. Biochem J. 2008; 409: 581-589.

49. Bermúdez-Lugo JA, Perez-Gonzalez O, RosalesHernández MC, Ilizaliturri-Flores I, Trujillo-Ferrara J, Correa-Basurto J. Exploration of the valproic acid binding site on histone deacetylase 8 using docking and molecular dynamic simulations. J Mol Model. 2012; 18: 2301-2310. 
50. Olsen EA, Kim YH, Kuzel TM, Pacheco TR, Foss FM, Parker $S$ et al. Phase Ilb multicenter trial of vorinostat in patients with persistent, progressive, or treatment refractory cutaneous T-cell lymphoma. J Clin Oncol. 2007; 25: 3109-3115.

51. Kirschbaum $\mathrm{MH}$. Histone deacetylase inhibitors and Hodgkin's lymphoma. Lancet Oncol. 2011; 12813): 1178-1179.

52. Lane AA, Chabner BA. Histone deacetylase inhibitors in cancer therapy. 2009; 27 (32): 5459-5468.

53. Münster $P$, Marchion D, Bicaku E, Lacevic M, Kim J, Centeno B et al. Clinical and biological effects of valproic acid as a histone deacetylase inhibitor on tumor and surrogate tissues: phase I/II trial of valproic acid and epirubicin/FEC. Clin Cancer Res. 2009; 15 (7): 2488-2496

54. Mohammed TA, Holen KD, Jaskula-Sztul R, Mulkerin DR, Lubner SJ, Schelman WR et al. A pilot phase II study of valproic acid for treatment of low-grade neuroendocrine carcinoma. Oncologist. 2011; 16 (6): 835-843.

55. Fenaux P, Mufti GJ, Hellstrom-Lindberg E, Santini V, Finelli C, Giagounidis A et al. Efficacy of azacitidine compared with that of conventional care regimens in the treatment of higher-risk myelodysplastic syndromes: a randomised, open-label, phase III study. Lancet Oncol. 2009; 10 (3): 223-232.

56. Fenaux P, Mufti GJ, Hellström-Lindberg E, Santini V, Gattermann N, Germing $U$ et al. Azacitidine prolongs overall survival compared with conventional care regimens in elderly patients with low bone marrow blast count acute myeloid leukemia. J Clin Oncol. 2010; 28 (4): 562-569.

57. Raffoux E, Cras A, Recher C, Boëlle PY, De Labarthe A, Turlure $P$ et al. Phase 2 clinical trial of 5 -azacitidine, valproic acid, and all-trans retinoic acid in patients with high-risk acute myeloid leukemia or myelodysplastic syndrome. Oncotarget. 2010; 1 (1): 34-42

58. Münster P, Marchion D, Bicaku E, Schmitt M, Lee JH, DeConti $\mathrm{R}$ et al. Phase I trial of histone deacetylase inhibition by valproic acid followed by the topoisomerase II inhibitor epirubicin in advanced solid tumors: a clinical and translational study. J Clin Oncol. 2007; 25 (15): 1979-1985.
59. Daud AI, Dawson J, DeConti RC, Bicaku E, Marchion D, Bastien $S$ et al. Potentiation of a topoisomerase I inhibitor, karenitecin, by the histone deacetylase inhibitor valproic acid in melanoma: translational and phase I/II clinical trial. Clin Cancer Res. 2009; 15 (7): 2479-2487.

60. Coronel J, Cetina L, Pacheco I, Trejo-Becerril C, González-Fierro A, De la Cruz-Hernández E et al. A double-blind, placebo-controlled, randomized phase III trial of chemotherapy plus epigenetic therapy with hydralazine valproate for advanced cervical cancer. Preliminary results. Med Oncol. 2011; 28 (1): S540S546.

61. Kang H, Gillespie TW, Goodman M, Brodie SA, Brandes $M$, Ribeiro $M$ et al. Long-term use of valproic acid in US veterans is associated with a reduced risk of smoking-related cases of head and neck cancer. Cancer. 2014; 120 (6): 1394-1400.

62. Dueñas-Gonzalez A, Coronel J, Cetina L, GonzálezFierro A, Chavez-Blanco A, Taja-Chayeb L. Hydralazine-valproate: a repositioned drug combination for the epigenetic therapy of cancer. 2014; 10 (10): 1433-1444.

63. Luna-Palencia GR, Martínez-Ramos F, Vásquez-Moctezuma I, Fragoso-Vázquez MJ, Mendieta-Wejebe JE, Padilla-Martínez II et al. Three amino acid derivatives of valproic acid: design, synthesis, theoretical and experimental evaluation as anticancer agents. Anticancer Agents Med Chem. 2014; 14 (7): 984-993.

64. Prestegui-Martel B, Bermúdez-Lugo JA, Chávez-Blanco A, Dueñas-González A, García-Sánchez JR, PérezGonzález OA et al. N-(2-hydroxyphenyl)-2-propylpentanamide, a valproic acid aryl derivative designed in silico with improved anti-proliferative activity in HeLa, rhabdomyosarcoma and breast cancer cells. J Enzyme Inhib Med Chem. 2016; 31 (3): 140-149.

65. Luna-Palencia GR, Correa-Basurto J, Trujillo-Ferrara J, Meraz-Ríos MA, Vásquez-Moctezuma I. Epigenetic evaluation of $\mathrm{N}$-(2-hydroxyphenyl)-2-Propylpentanamide, a valproic acid aryl derivative with activity against hela cells. Curr Mol Pharmacol. 2020; 13: 1. doi.org/10.2174/187446721366620 0730113828 . 